\title{
On the Dirichlet Problem to Elliptic Equation, the Order of which Degenerates at the Axis of a Cylinder
}

\author{
Stasys Rutkauskas \\ Institute of Mathematics and Informatics of Vilnius University \\ Akademijos str. 4, LT-08863 Vilnius, Lithuania \\ E-mail: stasys.rutkauskas@mii.vu.lt
}

Received November 25, 2016; revised July 25, 2017; published online September 15, 2017

\begin{abstract}
In this article, an elliptic equation, which type degenerates (either weakly or strongly) at the axis of 3-dimensional cylinder, is considered. The statement of a Dirichlet type problem in the class of smooth functions is given and, subject to the type of degeneracy, the classical solutions are composed. The uniqueness of the solutions is proved and the continuity of the solutions on the line of degeneracy is discussed.
\end{abstract}

Keywords: boundary value problems, degenerate, elliptic equation, second-order equation.

AMS Subject Classification: $35 \mathrm{~J} 70$.

\section{Introduction and statement of the problem}

In the cylinder $Q=\left\{(x, y, z): x^{2}+y^{2}<\rho^{2}, 0<z<h\right\}$, we consider the equation

$$
r^{2 \alpha}\left(u_{x x}+u_{y y}+u_{z z}\right)-c u=0, \quad \alpha>0,
$$

where $r=\sqrt{x^{2}+y^{2}}, c>0$ is a real constant. Evidently, equation (1.1) is elliptic outside of the line $x=y=0$ and its order degenerates at this line, i.e. at the axis of cylinder $Q$. If $\alpha \leq 1$, then the type of degeneracy of Eq. (1.1) is called to be regular (or weak), and in the case $\alpha>1$ it called to be irregular (or strong) [14].

The Diriclet type problems for the elliptic systems, which are irregularly degenerate at the inner point of a considered domain, are developed, e.g., in $[2,7,8]$. In $[9,10,11]$, the Dirichlet problem is considered for equation

$$
u_{z z}+r^{2 \alpha}\left(u_{x x}+u_{y y}\right)-c u=0, \quad \alpha>0,
$$

degeneracy of which is the other than in the case of Eq. (1.1), i.e. the type of Eq. (1.2) degenerates at $z$-axis. There is shown here that, under zero boundary value conditions on the bases of cylinder $Q$, this problem has the unique solution 
from the class $C^{2}\left(Q_{0}\right) \cap C(\bar{Q})$, and, in the case of the general boundary value conditions, the well-posedness of Dirichlet problem and the continuity of the solution on the line $x=y=0$ is related with the behavior of the boundary functions in the vicinity of the points $P_{0}(0,0,0)$ and $P_{h}(0,0, h)$, in which this line crosses the bases of cylinder $Q$. Also there is shown that, in particular case $\alpha=1$, the solution of this problem is non-continuous on the liene $x=y=0$, if the boundary value conditions are non-zero on the bases of cylinder $Q$.

In this paper, we discuss the Dirichlet type problem to Eq. (1.1). We shall show up here that, under the zero boundary value conditions on both bases of cylinder $Q$, this problem has the unique solution for all $\alpha>1$ from the same class $C^{2}\left(Q_{0}\right) \cap C(\bar{Q})$ as in the case of Eq. (1.2). If the boundary value conditions are non-zero, in the case $\alpha=1$, we shall show that the solution can be not only non-continuous (differently than in the case of Eq. (1.2), but also continuous on the line of the degeneracy. Specifically, we obtain the sufficient conditions of the continuity, which are related with the behavior of the boundary functions at both points $P_{0}, P_{h}$ and also with the coefficient $c$.

It is convenient to introduce the cylindrical coordinates $r, \varphi, z(|\varphi| \leq \pi)$ in which an Eq. (1.1) takes the form

$$
u_{z z}+L(u)=0
$$

where operator $L$ is defined by

$$
L=\frac{1}{r} \frac{\partial}{\partial r}\left(r \frac{\partial}{\partial r}\right)+\frac{1}{r^{2}} \frac{\partial^{2}}{\partial \varphi^{2}}-r^{-2 \alpha} c .
$$

(Here we denote a solution $v(r, \varphi, z)=u(r \cos \varphi, r \sin \varphi, z)$ of Eq. (1.3) by $u(r, \varphi, z)$ again.)

In these coordinates, $Q=\{(r, \varphi, z):(r, \varphi) \in \Delta, 0<z<h\}$, where $\Delta$ is the disk $\{(r, \varphi): r<\rho,|\varphi| \leq \pi\}$. Besides, we use the following denotations: $K=\partial \Delta$ is the circle, $S=K \times[0, h]$ is the lateral surface and $B_{i}=\{(r, \varphi, z)$ : $(r, \varphi) \in \bar{\Delta}, z=(i-1) h\}, i=1,2$, are the bases of cylinder $Q, \Delta_{\delta}=\{(r, \varphi): \delta<$ $r<\rho,|\varphi| \leq \pi\}, \delta \geq 0$, is the ring, $Q_{\delta}=\left\{(r, \varphi, z):(r, \varphi) \in \Delta_{\delta}, 0<z<h\right\}$ is cylindrical ring, $B_{i \delta}=\left\{(r, \varphi, z):(r, \varphi) \in \bar{\Delta}_{\delta}, z=(i-1) h\right\}, i=1,2$, are the bases of the cylindrical ring $Q_{\delta}, \Omega$ is the domain $\{(\varphi, z):|\varphi| \leq \pi, 0 \leq z \leq h\}$. As usually, we denote by $\bar{D}$ the closure of any domain $D$, by $\mathbb{N}$ and $\mathbb{N}_{0}$ the set of natural numbers and the set of non-negative integer numbers, correspondingly, and by $C^{l}(D)$ the class of functions which derivatives are continuous trough up to the order $l$ in a domain $D$.

The object of this work is the following Dirichlet problem.

Problem D. Find the solution $u(r, \varphi, z)$ of Eq. (1.3) in the class of functions $C^{2}\left(Q_{0}\right) \cap C\left(\bar{Q} \backslash\{r=0\}\right.$ ) (or, maybe, in the class $C^{2}\left(Q_{0}\right) \cap C(\bar{Q})$, which is bounded in $Q_{0}$ and satisfies the boundary value conditions

$$
u(\rho, \varphi, z)=f(\varphi, z)
$$

for $(\varphi, z) \in \Omega$ and

$$
u(r, \varphi,(i-1) h)=f_{i}(r, \varphi), \quad i=1,2,
$$


for $(r, \varphi) \in \Delta_{0} \cup K$ (or, maybe, for $(r, \varphi) \in \bar{\Delta}$, where $f$ and $f_{i}$ are given continuous and $2 \pi$-periodic in $\varphi$ functions such that

$$
f(\varphi,(i-1) h)=f_{i}(\rho, \varphi), \quad i=1,2 .
$$

The particular case of Problem D, when $f_{i}(r, \varphi) \equiv 0, i=1,2$, in the disk $\Delta$, we shall call as Problem D0. Obviously, in this case, condition (1.5) takes the shape

$$
u(r, \varphi,(i-1) h)=0, \quad i=1,2 .
$$

If compatibility condition (1.6) is replaced by

$$
f(\varphi,(i-1) h)=f_{i}(\rho, \varphi)=0, \quad i=1,2,
$$

such partial case of Problem D we call as Problem D1.

\section{Solutions of Problem D0}

Primarily, we indicate the maximum principle for the solutions of Problem D.

Lemma 1. If the solution $u \in C^{2}\left(Q_{0}\right) \cap C(\bar{Q} \backslash\{r=0\})$ of Problem D does not attain neither positive maximum nor negative minimum on the line $r=0$, then there holds the estimate

$$
|u(r, \varphi, z)|<\max \left\{\max _{\Omega}|f(\varphi, z)|, \max _{i=1,2} \max _{\bar{\Delta}}\left|f_{i}(r, \varphi)\right|\right\}, \quad \forall(r, \varphi, z) \in Q_{0} .
$$

Proof. Due to the ellipticity of Eq. (1.3) in $Q_{0}$ and in view of inequality $c>0$, any solution of this equation attains neither positive maximum nor negative minimum in $Q_{0}[1]$. Then, under the assumption of the Lemma, the solution $u \in C^{2}\left(Q_{0}\right) \cap C(\bar{Q} \backslash\{r=0\})$ of Problem D attains either positive maximum or negative minimum only on the surface of cylinder $Q_{0}$. Therefore, estimate (1.7) holds because of (1.4), (1.5).

By the separation of variables, we obtain the following partial solutions

$$
u_{m}(r, \varphi, z ; \gamma)=R_{m}(r ; \gamma) Z(z ; \gamma) \begin{cases}\cos m \varphi, & m \in \mathbb{N} \\ \sin m \varphi, & m \in \mathbb{N}_{0}\end{cases}
$$

of Eq. (1.3), where $Z(z ; \gamma)$ and $R_{m}(r ; \gamma)$ are the solutions of corresponding equations

$$
\begin{aligned}
& Z^{\prime \prime}+\gamma Z=0 \\
& r \frac{\mathrm{d}}{\mathrm{d} r}\left(r \frac{\mathrm{d} R}{\mathrm{~d} r}\right)-\left(m^{2}+\gamma r^{2}+c r^{2(1-\alpha)}\right) R=0
\end{aligned}
$$

Setting in (2.1) and (2.2)

$$
\gamma=\gamma_{n}^{2}:=(n \pi / h)^{2}
$$


we get the sequence of partial solutions

$$
u_{m n}(r, \varphi, z)=R_{m n}(r) \sin \gamma_{n} z \begin{cases}\cos m \varphi, & m, n \in \mathbb{N}, \\ \sin m \varphi, & m \in \mathbb{N}_{0}, n \in \mathbb{N}\end{cases}
$$

of Eq. (1.3), where $R_{m n}(r):=R_{m}\left(r ; \gamma_{n}^{2}\right)$. Evidently, these partial solutions satisfy condition (1.5).

According to the statement of Problem D, we take interest only in the bounded in $Q_{0}$ solutions of Eq. (1.3). Thus, the function $R_{m n}$ in (2.3) ought to be bounded at the point $r=0$. So we are in need to investigate the behavior of the solutions of the equation

$$
r \frac{\mathrm{d}}{\mathrm{d} r}\left(r \frac{\mathrm{d} R}{\mathrm{~d} r}\right)-\left(m^{2}+\gamma_{n}^{2} r^{2}+c r^{2(1-\alpha)}\right) R=0
$$

at the point $r=0$. If $\alpha \neq 1$, then the solutions of Eq. (2.4) can not be expressed analytically by elementary or transcendental functions. However, in all cases, one can give the asymptotics of linear independent solutions of this equation.

Let $\alpha<1$. In this case, there exist the solutions $R_{m n}^{(1)}$ and $R_{m n}^{(2)}$ such that

$$
R_{m n}^{(1)}(r)=r^{m}[1+o(1)], m \in \mathbb{N}_{0}, \quad R_{m n}^{(2)}(r)= \begin{cases}r^{-m}[1+o(1)], & m \in \mathbb{N} \\ \ln r[1+o(1)], & m=0\end{cases}
$$

as $r \rightarrow 0[6]$.

If $\alpha=1$, then Eq. (2.4) represents so called Bessel equation, which linear independent solutions $R_{m n}^{(3)}$ and $R_{m n}^{(4)}$ are of the shape

$$
R_{m n}^{(3)}(r)=I_{\sqrt{m^{2}+c}}\left(\gamma_{n} r\right), \quad R_{m n}^{(4)}(r)=K_{\sqrt{m^{2}+c}}\left(\gamma_{n} r\right),
$$

here $I_{\sqrt{m^{2}+c}}$ and $K_{\sqrt{m^{2}+c}}$ are the modified Bessel functions of the first and second kind, respectively. It is well known that $[3,13]$

$$
R_{m n}^{(3)}(r)=c_{m n}^{(1)} r^{\sqrt{m^{2}+c}}\left[1+O\left(r^{2}\right)\right], \quad R_{m n}^{(4)}(r)=c_{m n}^{(2)} r^{-\sqrt{m^{2}+c}}[1+o(1)]
$$

as $r \rightarrow 0$. (Here $c_{m n}^{(1)}$ and $c_{m n}^{(2)}$ are some non-zero constants, which can be calculated exactly.) Thus, only the solution $R_{m n}^{(3)}$ is bounded at the point $r=0$. Specifically, $\lim _{r \rightarrow 0} R_{m n}^{(3)}(r)=0$ for all $m \in \mathbb{N}_{0}$ and for all $n \in \mathbb{N}$.

Assume that $\alpha>1$. According to Eq. (2.4), the function

$$
P=\sqrt{r} R
$$

satisfies the equation

$$
P^{\prime \prime}-\left[c r^{-2 \alpha}+g_{m n}(r)\right] P=0,
$$

where $g_{m n}(r)=\left(m^{2}-1 / 4\right) r^{-2}+\gamma_{n}^{2}$. Due to the evident condition

$$
\int_{0}^{\rho} r^{\alpha}\left|g_{m n}(r)\right| \mathrm{d} r<\infty, \quad \alpha>1
$$


the main parts of asymptotic of the solutions of Eq. (2.8) do not depend of function $g_{m n}$ as $r \rightarrow 0$ [5]. Namely, there exist the linear independent solutions $P_{m n}^{(1)}$ and $P_{m n}^{(2)}$ such that

$$
\begin{aligned}
& P_{m n}^{(1)}(r)=r^{\alpha / 2} \exp \left\{-\frac{\sqrt{c}}{\alpha-1} r^{1-\alpha}\right\}\left[1+O\left(r^{\alpha-1}\right)\right], \\
& P_{m n}^{(2)}(r)=r^{\alpha / 2} \exp \left\{\frac{\sqrt{c}}{\alpha-1} r^{1-\alpha}\right\}[1+o(1)]
\end{aligned}
$$

as $r \rightarrow 0$ for all $m \in \mathbb{N}_{0}$ and for all $n \in \mathbb{N}[5,6]$. Jointly with (2.7), this yields the existence of linear independent solutions

$$
R_{m n}^{(5)}(r)=r^{-1 / 2} P_{m n}^{(1)}(r), \quad R_{m n}^{(6)}(r)=r^{-1 / 2} P_{m n}^{(2)}(r)
$$

of Eq. (2.4) with the asymptotic expansions:

$$
\begin{aligned}
& R_{m n}^{(5)}(r)=r^{(\alpha-1) / 2} \exp \left\{-\frac{\sqrt{c}}{\alpha-1} r^{1-\alpha}\right\}\left[1+O\left(r^{\alpha-1}\right)\right] \\
& R_{m n}^{(6)}(r)=r^{(\alpha-1) / 2} \exp \left\{\frac{\sqrt{c}}{\alpha-1} r^{1-\alpha}\right\}[1+o(1)]
\end{aligned}
$$

as $r \rightarrow 0$. Thus, $\lim _{r \rightarrow 0} R_{m n}^{(5)}(r)=0$ and $\lim _{r \rightarrow 0} R_{m n}^{(6)}(r)=\infty$ for all $m \in \mathbb{N}_{0}$ and for all $n \in \mathbb{N}$.

Let us note that the solutions $R_{m n}^{(1)}(r)$ and $R_{m n}^{(5)}(r)$ are one-valued by $(2.5)$ and (2.10), respectively. There holds the following lemma.

Lemma 2. The solutions $R_{m n}^{(j)}(r), j=1,3,5$, of Eq. (2.4) are monotonously increasing for all $m \in \mathbb{N}_{0}$ and for $n \in \mathbb{N}$.

Proof. Let us note that the solutions of Eq. (2.4) cannot attain neither positive maximum nor negative minimum on the interval $(0,+\infty)$. Then monotonously increase of the solutions $R_{m n}^{(1)}(r)$ for $m, n \in \mathbb{N}$ and $R_{m n}^{(j)}(r), j=3,5$, for $m \in \mathbb{N}_{0}$ and $n \in \mathbb{N}$ follows immediately from asymptotic expressions (2.5), (2.6) and (2.9), because the limit of these solutions is vanish for $r=0$.

Let us examine the solution $R_{0 n}^{(1)}, n \in \mathbb{N}$. It follows from (2.5) that $\lim _{r \rightarrow 0} R_{0 n}^{(1)}(r)=1$. Assume that this solution decreases on some interval $\left(0, r_{0}\right)$, where $r_{0}$ is thus small that $R_{0 n}^{(1)}(r)$ is positive on $\left(0, r_{0}\right]$, and it does not have any positive minimum on $\left(0, r_{0}\right)$. Then $R_{0 n}^{\prime}(r)<0, r \in\left(0, r_{0}\right]$. Further, since

$$
\frac{\mathrm{d}}{\mathrm{d} r}\left(r \frac{\mathrm{d} R_{0 n}^{(1)}}{\mathrm{d} r}\right) \equiv r\left(\gamma_{n}^{2}+c r^{-2 \alpha}\right) R_{0 n}^{(1)}>0, \quad r \in\left(0, r_{0}\right],
$$

the function $r R_{0 n}^{\prime}(r)$ is monotonously increasing on the interval $\left(0, r_{0}\right)$ and it is negative on $\left(0, r_{0}\right]$. Then there exists a constant $\mu>0$ such that

$$
\frac{\mathrm{d}}{\mathrm{d} r} R_{0 n}^{(1)}(r) \leq-\frac{\mu}{r}, \quad r \in\left(0, r_{0}\right]
$$


Integrating this inequality on the interval $\left(\varepsilon, r_{0}\right)$, we obtain that

$$
R_{0 n}^{(1)}(\varepsilon) \geq \mu \ln \frac{r_{0}}{\varepsilon}+R_{0 n}\left(r_{0}\right) ;
$$

consequently, $\lim _{\varepsilon \rightarrow 0} R_{0 n}(\varepsilon)=+\infty$, but this is in contradiction with the boundedness of the solution $R_{0 n}^{(1)}$ at the point $r=0$. Therefore, this solution is increasing in a neighbourhood of the point $r=0$. That yields the increase of $R_{0 n}^{(1)}$ on the interval $(0,+\infty)$, because the solutions of Eq. (1.3) cannot attain any positive maximum on the interval $(0,+\infty)$.

Assume that $f \in C^{2}(\Omega)$ and $f(\varphi,(i-1) h)=0, i=1,2$. Then function $f$ can be expanded into double Fourier series

$$
f(\varphi, z)=\sum_{n=1}^{\infty} a_{0 n} \sin \gamma_{n} z+\sum_{n=1}^{\infty} \sum_{m=1}^{\infty}\left(a_{m n} \cos m \varphi+b_{m n} \sin m \varphi\right) \sin \gamma_{n} z
$$

$(\varphi, z) \in \Omega$, with the coefficients

$$
\left.\begin{array}{l}
a_{m n} \\
b_{m n}
\end{array}\right\}=\frac{\kappa_{m n}}{\pi^{2}} \int_{0}^{h} \sin \gamma_{n} z \mathrm{~d} z \int_{-\pi}^{\pi} f(\varphi, z)\left\{\begin{array}{c}
\cos m \varphi \\
\sin m \varphi
\end{array}\right\} \mathrm{d} \varphi
$$

where $\kappa_{00}=1$ and $\kappa_{0 n}=\frac{1}{2}, \kappa_{m n}=\frac{1}{4}$ for $m, n \in \mathbb{N}$. Since $f \in C^{2}(\Omega)$, series (2.11) converges uniformly and absolutely on $\Omega$ [4].

Introduce the series

$$
\begin{aligned}
& u_{0}^{(j)}(r, \varphi, z)=\sum_{n=1}^{\infty} \frac{R_{0 n}^{(j)}(r)}{R_{0 n}^{(j)}(\rho)} a_{0 n} \sin \gamma_{n} z \\
& \quad+\sum_{n=1}^{\infty} \sum_{m=0}^{\infty} \frac{R_{m n}^{(j)}(r)}{R_{m n}^{(j)}(\rho)}\left(a_{m n} \cos m \varphi+b_{m n} \sin m \varphi\right) \sin \gamma_{n} z, \quad j=1,3,5,
\end{aligned}
$$

which represent the composition of partial solutions of Eq. (1.3), evidently.

By virtue of Lemma 1,

$$
0<R_{0 n}^{(j)}(r) \leq R_{0 n}^{(j)}(\rho), \quad j=1,3,5,
$$

on $[0, \rho]$, hence

$$
\left|u_{0}^{(j)}(r, \varphi, z)\right|=\sum_{n=1}^{\infty}\left|a_{0 n}\right|+\sum_{n=1}^{\infty} \sum_{m=0}^{\infty}\left(\left|a_{m n}\right|+\left|b_{m n}\right|\right)<\infty, \quad j=1,3,5,
$$

for each $(r, \varphi, z) \in \bar{Q}$ because of absolutely convergence of series (2.11). Therefore, series (2.13) converge uniformly and absolutely everywhere in cylinder $\bar{Q}$ including the line of degeneracy $r=0$. Further, it is easily seen that

$$
\begin{aligned}
& u_{0}^{(j)}(\rho, \varphi, z)=f(\varphi, z) \quad \forall(\varphi, z) \in \Omega \\
& u_{0}^{(j)}(r, \varphi, 0)=u_{0}^{(j)}(r, \varphi, h)=0 \quad \forall(r, \varphi) \in \bar{\Delta}, j=1,3,5 .
\end{aligned}
$$


So by (2.13), boundary value conditions (1.4) and (1.5) are satisfied. Due to the ellipticity of Eq. (1.3) in $Q_{0}$ and because of the maximum principle for elliptic equations, which holds in view of the condition $c>0$, the sums of series (2.13) are twice continuously differentiable and satisfy Eq. (1.3) in $Q_{0}$. Hence, by (2.13), we represent the solutions $u_{0}^{(1)}, u_{0}^{(3)}$ and $u_{0}^{(3)}$ of Problem D0 corresponding to the parameter $0<\alpha<1, \alpha=1$ and $\alpha>1$. that

Further, according to (2.5), (2.6), (2.10), we get immediately from (2.13)

$$
\lim _{r \rightarrow 0} u_{0}^{(j)}(r, \varphi, z)= \begin{cases}\sum_{n=0}^{\infty} \alpha_{0 n} \sin \gamma_{n} z, & \text { for } j=1, \\ 0, & \text { for } j=3,5\end{cases}
$$

Since

$$
\sum_{n=0}^{\infty} \alpha_{0 n} \sin \gamma_{n} z=\frac{1}{2 \pi} \int_{-\pi}^{\pi} f(\varphi, z) \mathrm{d} \varphi,
$$

(because of definition (2.12) of the coefficients $\alpha_{0 n}$ ), this yields the estimate

$$
\left|u_{0}^{(1)}(0, \varphi, z)\right| \leq \frac{1}{2 \pi} \int_{-\pi}^{\pi}|f(\varphi, z)| \mathrm{d} \varphi \leq \max _{\Omega}|f(\varphi, z)| .
$$

So all solutions $u_{0}^{(j)}, j=1,3,5$, do not attain neither positive maximum nor negative minimum on the line $r=0$. Thus, it follows from Lemma 1 that

$$
\left|u_{0}^{(j)}(r, \varphi, z)\right|<\max _{\Omega}|f(\varphi, z)|, \quad j=1,3,5
$$

everywhere in $Q_{0}$. This yields the uniqueness of the obtained solutions of Problem D0.

It follows from above the following theorem.

Theorem 1. Let $f \in C^{2}(\Omega)$ and let $f(\varphi,(i-1) h)=0, i=1,2$. Then Problem D0 has the unique solution from the class $C^{2}\left(Q_{0}\right) \cap C(\bar{Q})$. Subject to the type of the degeneracy of Eq. (1.3), the solution of this problem can be expressed by series (2.13).

\section{Solution of Problem D1 (the case $\alpha=1$ )}

Next we consider only the case of the operator $L$, when $\alpha=1$. Preliminary, we deal with the following eigenvalues problem.

EV-Problem. Find the solutions $w(r, \varphi ; \gamma)$ of equation

$$
L(w):=\frac{1}{r} \frac{\partial}{\partial r}\left(r \frac{\partial w}{\partial r}\right)+\frac{1}{r^{2}} \frac{\partial^{2} w}{\partial \varphi^{2}}-\frac{c}{r^{2}} w=-\gamma w
$$

in the class of functions $C^{2}\left(\Delta_{0} \cup K\right)$, which are bounded in $\Delta_{0}$, also are $2 \pi$ periodic in $\varphi$ and satisfy the boundary value condition

$$
w(\rho, \varphi ; \gamma)=0
$$


Using the method of separate variables, we obtain the following partial solutions of Eq. (3.1):

$$
R_{m}(r ; \gamma) \times \begin{cases}\cos m \varphi, & m \in \mathbb{N}_{0} \\ \sin m \varphi, & m \in \mathbb{N}\end{cases}
$$

where $R_{m}(r ; \gamma)$ is the non-trivial solution of Sturm-Liouville problem (next we call it SL-Problem)

$$
\begin{aligned}
& \frac{1}{r} \frac{\mathrm{d}}{\mathrm{d} r}\left(r \frac{\mathrm{d} R}{\mathrm{~d} r}\right)-\frac{m^{2}+c}{r^{2}} R=-\gamma R, \\
& R(\rho)=0, \quad|R(r)|<\infty \quad \text { on }(0, \rho] .
\end{aligned}
$$

Eq. (3.3) has only one bounded solution

$$
R_{m}(r ; \gamma)=J_{\sqrt{m^{2}+c}}(\gamma r)
$$

with the accuracy of constant multiplier, whereas all other linear independent solutions are unbounded at the point $r=0$. (Here $J_{\nu}$ is Bessel function of the first kind of order $\nu[13]$.) Let

$$
\lambda_{m 0}<\lambda_{m 1}<\cdots<\lambda_{m n}<\cdots
$$

be the positive roots of Bessel function $J_{\sqrt{m^{2}+c}}$. Choose in Eq. (3.1) the values of the parameter $\gamma$ by the definition

$$
\gamma_{m n}=\lambda_{m n} / \rho, \quad n \in \mathbb{N}_{0} .
$$

Then Bessel functions $J_{\sqrt{m^{2}+c}}\left(\lambda_{m n} r / \rho\right), n \in \mathbb{N}_{0}$ represent the set of the eigenfunctions of SL-Problem (3.3), (3.4), which are continuous at the point $r=0$ because of the asymptotic expansion $[3,13]$

$$
J_{\sqrt{m^{2}+c}}\left(\lambda_{m n} \frac{r}{\rho}\right)=\left(\frac{\lambda_{m n}}{2 \rho}\right)^{\sqrt{m^{2}+c}} r^{\sqrt{m^{2}+c}}(1+O(r))
$$

as $r \rightarrow 0$. So we get the following sequence of the eigenfunctions of EV-Problem $(3.1),(3.2))$ :

$$
\begin{aligned}
w_{m n}(r, \varphi) & :=w\left(r, \varphi ; \lambda_{m n} / \rho\right) \\
& =J_{\sqrt{m^{2}+c}}\left(\frac{\lambda_{m n}}{\rho} r\right) \times \begin{cases}\cos m \varphi, & m, n \in \mathbb{N}_{0}, \\
\sin m \varphi, & m \in \mathbb{N}, n \in \mathbb{N}_{0} .\end{cases}
\end{aligned}
$$

According to $(3.6), w_{m n}(0, \varphi)=0$ and $w_{m n} \in C^{2}\left(\Delta_{0}\right) \cap C(\bar{\Delta})$.

Next we shall deal with the conditions under which the boundary functions $f_{i}(r, \varphi), i=1,2$, from (1.5) can be expressed in the defined by (3.7) eigenfuntions $w_{m n}$ of EV-Problem (3.1), (3.2).

Lemma 3. Assume that functions $f_{i}, i=1,2$, are such that 
(i) $f_{i}$ and $\frac{\partial f_{i}}{\partial \varphi} \in C(\bar{\Delta})$;

(ii) $f_{i}(\rho, \varphi)=0$ for all $\varphi \in[-\pi, \pi], \frac{\partial f_{i}}{\partial r} \in C\left(\Delta_{0} \cup K\right)$ and

$$
\int_{0}^{\rho}\left|\frac{\partial f_{i}(r, \varphi)}{\partial r}\right| \mathrm{d} r<\infty, \quad \forall \varphi \in[-\pi, \pi] .
$$

Then, under conditions (i), (ii), each function $f_{i}$ can be expanded into the series

$$
\begin{aligned}
f_{i}(r, \varphi)= & \frac{1}{2} \sum_{n=0}^{\infty} a_{0 n} J_{\sqrt{c}}\left(\frac{\lambda_{0 n}}{\rho} r\right) \\
& +\sum_{m=1}^{\infty} \sum_{n=0}^{\infty} J_{\sqrt{m^{2}+c}}\left(\frac{\lambda_{m n}}{\rho} r\right)\left(a_{m n}^{(i)} \cos m \varphi+b_{m n}^{(i)} \sin m \varphi\right)
\end{aligned}
$$

where

$$
\begin{aligned}
& \left.\begin{array}{l}
a_{m n}^{(i)} \\
b_{m n}^{(i)}
\end{array}\right\}=\frac{2}{\pi \rho^{2} J_{1+\sqrt{m^{2}+c}}^{2}\left(\lambda_{m n}\right)} \\
& \times \int_{0}^{\rho} J_{\sqrt{m^{2}+c}}\left(\frac{\lambda_{m n}}{\rho} r\right) r \mathrm{~d} r \int_{-\pi}^{\pi} f_{i}(r, \varphi)\left\{\begin{array}{l}
\cos m \varphi \\
\sin m \varphi
\end{array}\right\} \mathrm{d} \varphi .
\end{aligned}
$$

These series converge uniformly in the ring $\bar{\Delta}_{\delta}$ and converge uniformly and absolutely in the ring $\bar{\Delta}_{\delta} \backslash \Delta_{\rho-\delta}$ for every $\delta \in(0, \rho)$.

Let, besides (i) and (ii), there hold conditions

(iii) $f_{i}(0, \varphi)=0$ for all $\varphi \in[-\pi, \pi]$;

(iv) $\frac{\partial f_{i}}{\partial r} \in C(\bar{\Delta}), \frac{\partial^{2} f_{i}}{\partial r^{2}} \in C\left(\Delta_{0} \cup K\right)$, also $\frac{\partial f_{i}(r, \varphi)}{\partial r}=o(1)$ and $\frac{\partial^{2} f_{i}(r, \varphi)}{\partial r^{2}}=O(1)$ uniformly with respect to $\varphi$ as $r \rightarrow 0$.

Then series (3.8) converges uniformly and absolutely in the whole disk $\bar{\Delta}$.

Proof. Under condition (i), one can expand both function $f_{i}(r, \varphi), i=1,2$, by uniformly and absolutely converging in $\bar{\Delta}$ Fourier series [12]

$$
f_{i}(r, \varphi)=\frac{1}{2} a_{0}^{(i)}(r)+\sum_{m=1}^{\infty}\left(a_{m}^{(i)}(r) \cos m \varphi+b_{m}^{(i)}(r) \sin m \varphi\right)
$$

where

$$
\left.\begin{array}{l}
a_{m}^{(i)}(r) \\
b_{m}^{(i)}(r)
\end{array}\right\}=\frac{1}{\pi} \int_{-\pi}^{\pi} f_{i}(r, \varphi)\left\{\begin{array}{l}
\cos m \varphi \\
\sin m \varphi
\end{array}\right\} \mathrm{d} \varphi, \quad \begin{aligned}
& m \in \mathbb{N}_{0} \\
& m \in \mathbb{N}
\end{aligned}
$$

Obviously, if we want have the expansion of the functions $f_{i}(r, \varphi)$ by the eigenfunctions $w_{m n}$ of EV-Problem, then there is suffice to expand the coefficients $a_{m}^{(i)}(r)$ and $b_{m}^{(i)}(r), i=1,2$, of series (3.10) in the eigenfunctions of SL-Problem (3.4), (3.5). 
Let conditions (i) and (ii) hold. Then it follows from (3.11) that

$$
a_{m}^{(i)}(\rho)=0, \quad a_{m}^{(i)} \in C[0, \rho], \quad \frac{\mathrm{d} a_{m}}{\mathrm{~d} r} \in C(0, \rho]
$$

and

$$
\begin{aligned}
\int_{0}^{\rho}\left|\frac{\mathrm{d} a_{m}^{(i)}(r)}{\mathrm{d} r}\right| \mathrm{d} t & =\frac{1}{\pi} \int_{0}^{\rho}\left|\int_{-\pi}^{\pi} \frac{\partial g(r, \varphi)}{\partial r} \cos m \varphi \mathrm{d} \varphi\right| \mathrm{d} r \\
& \leq \frac{1}{\pi} \int_{-\pi}^{\pi} \mathrm{d} \varphi \int_{0}^{\rho}\left|\frac{\partial g(r, \varphi)}{\partial r}\right| \mathrm{d} r<\infty
\end{aligned}
$$

These properties of functions $a_{m}^{(i)}(r), i=1,2$, are sufficient in order to expand them into Fourier-Bessel series

$$
a_{m}^{(i)}(r)=\sum_{n=0}^{\infty} a_{m n}^{(i)} J_{\sqrt{m^{2}+c}}\left(\frac{\lambda_{m n}}{\rho} r\right), \quad m \in \mathbb{N}_{0},
$$

where

$$
a_{m n}^{(i)}=\frac{2}{\rho^{2} J_{\sqrt{m^{2}+c}+1}^{2}\left(\lambda_{m n}\right)} \int_{0}^{\rho} a_{m}^{(i)}(r) J_{\sqrt{m^{2}+c}}\left(\frac{\lambda_{m n}}{\rho} r\right) r \mathrm{~d} r .
$$

These series converge uniformly on each interval $[\delta, \rho]$ and converge uniformly and absolutely on each interval $[\delta, \rho-\delta]$ for every $\delta \in(0, \rho)($ see $[12,13])$.

Let all conditions (i)-(iv) of the lemma hold. Then we obtain from (3.11) that

$$
\begin{aligned}
& a_{m}^{(i)} \in C^{1}([0, \rho]), \quad a_{m}^{(i)}(0)=a_{m}^{(i)}(\rho)=\frac{\mathrm{d} a_{m}^{(i)}(0)}{\mathrm{d} r}=0, \\
& \left|\frac{\mathrm{d}^{2} a_{m}^{(i)}(r)}{\mathrm{d} r^{2}}\right|<\infty \quad \forall r \in(0, \rho), i=1,2 .
\end{aligned}
$$

This additional collection of the properties of functions $a_{m}^{(i)}, i=1,2$, yields the possibility to expand these functions into series (3.12), which converge uniformly and absolutely on the whole interval $[0, \rho]$ (see [12]).

Further, replacing in equality $(3.13)$ the functions $a_{m}^{(i)}(r), i=1,2$, by their expressions (3.11), we get for the coefficients $a_{m n}^{(i)}, i=1,2$, definition (3.9).

Just under the same conditions as in the case of the functions $a_{m n}^{(i)}$, in the same way as above, we obtain the expansions

$$
b_{m}^{(i)}(r)=\sum_{n=0}^{\infty} b_{m n}^{(i)} J_{\sqrt{m^{2}+c}}\left(\frac{\lambda_{m n}}{\rho} r\right), \quad i=1,2, m \in \mathbb{N}
$$

with the coefficients $b_{m n}^{(i)}$ defined by (3.9). Subject to the conditions of the Lemma, the properties of the convergence of series (3.14) are analogous as in the case of series (3.12). 
Finally, putting (3.12) and (3.14) into (3.10), we obtain representations (3.9). Moreover, it follows from the above the uniform convergence of series (3.9) in every ring $\bar{\Delta}_{\delta}$ and uniform and absolute convergence in every $\bar{\Delta}_{\delta} \backslash \Delta_{\rho-\delta}$ under conditions (i), (ii), and the uniform and absolute convergence in whole disk $\bar{\Delta}$ under conditions (i)-(iv).

Remark. Even though functions $f_{i}, i=1,2$, are such that $f_{i}(0, \varphi)=0$, conditions (i)-(ii) of Lemma 3 do not warrant entirely the uniformly convergence of series (3.12) and (3.14) on the whole interval $[0, \rho]$. That is the reason why, in the case of conditions (i)-(ii), we do not require for the vanishing of the both functions $f_{i}$ for $r=0$.

Remembering that $\gamma_{m n}$ is defined by (3.6), denote by $Z_{m n}^{(i)}(z), i=1,2$, the solutions of Eq. (2.1) with $\gamma=-\gamma_{m n}$, which satisfy the boundary value conditions

$$
Z_{m n}^{(1)}(0)=a_{m n}^{(1)}, \quad Z_{m n}^{(1)}(h)=a_{m n}^{(2)} ; \quad Z_{m n}^{(2)}(0)=b_{m n}^{(1)}, \quad Z_{m n}^{(2)}(h)=b_{m n}^{(2)},
$$

where $a_{m n}^{(i)}$ and $b_{m n}^{(i)}, i=1,2$, are given by (3.9). It is easily seen that those solutions are as follows:

$$
\begin{aligned}
& Z_{m n}^{(1)}(z)=\sinh ^{-1} \sqrt{\gamma_{m n}} h\left(a_{m n}^{(1)} \sinh \sqrt{\gamma_{m n}}(h-z)+a_{m n}^{(2)} \sinh \sqrt{\gamma_{m n}} z\right) \\
& Z_{m n}^{(2)}(z)=\sinh ^{-1} \sqrt{\gamma_{m n}} h\left(b_{m n}^{(1)} \sinh \sqrt{\gamma_{m n}}(h-z)+b_{m n}^{(2)} \sinh \sqrt{\gamma_{m n}} z\right) .
\end{aligned}
$$

So we obtain the following partial solutions

$$
J_{\sqrt{m^{2}+c}}\left(\frac{\lambda_{m n}}{\rho} r\right) \times \begin{cases}Z_{m n}^{(1)}(z) \cos m \varphi, & m, n \in \mathbb{N}_{0}, \\ Z_{m n}^{(2)}(z) \sin m \varphi, & m \in \mathbb{N}, n \in \mathbb{N}_{0}\end{cases}
$$

of Eq. (1.3). Evidently, they are continuous in $\bar{Q}$.

Let us compose the series

$$
\begin{aligned}
u_{1}(r, \varphi, z)= & \frac{1}{2} \sum_{n=0}^{\infty} J_{\sqrt{c}}\left(\frac{\lambda_{m n}}{\rho} r\right) Z_{0 n}^{(1)}(z) \\
& +\sum_{m=1}^{\infty} \sum_{n=0}^{\infty} J_{\sqrt{m^{2}+c}}\left(\frac{\lambda_{m n}}{\rho} r\right)\left(Z_{m n}^{(1)}(z) \cos m \varphi+Z_{m n}^{(2)}(z) \sin m \varphi\right) .
\end{aligned}
$$

In view of (3.15), this series coincides on the bases $B_{i}$ with the series (3.8) of the respective functions $f_{i}, i=1,2$. Observe also that $u_{1}(\rho, \varphi, z)=0$ because of $J_{\sqrt{m^{2}+c}}\left(\lambda_{m n}\right)=0$, i.e. series (3.16) converges (uniformly) on the surface $S$ of cylinder $Q$. Further, due to the obvious inequalities

$$
\left|Z_{m n}^{(1)}(z)\right| \leq\left|a_{m n}^{(1)}\right|+\left|a_{m n}^{(2)}\right|, \quad\left|Z_{m n}^{(2)}(z)\right| \leq\left|b_{m n}^{(1)}\right|+\left|b_{m n}^{(2)}\right|
$$

and in view of Lemma 4 , we get from (3.16) that

$$
\begin{aligned}
& \left|u_{1}(r, \varphi, z)\right| \leq \frac{1}{2} \sum_{n=0}^{\infty}\left(\left|a_{0 n}^{(1)}\right|+\left|a_{0 n}^{(2)}\right|\right)\left|J_{\sqrt{c}}\left(\frac{\lambda_{m n}}{\rho} r\right)\right| \\
& \quad+\sum_{m=1}^{\infty} \sum_{n=0}^{\infty}\left(\left|a_{m n}^{(1)}\right|+\left|a_{m n}^{(2)}\right|+\left|b_{m n}^{(1)}\right|+\left|b_{m n}^{(2)}\right|\right)\left|J_{\sqrt{m^{2}+c}}\left(\frac{\lambda_{m n}}{\rho} r\right)\right|<\infty
\end{aligned}
$$


in cylindrical ring $\bar{\Omega}_{\delta}, \delta \in(0, \rho)$, under conditions (i), (ii) and in $\bar{Q}$ under conditions (i)-(iv) of Lemma 3.

Thus, if functions $f_{i}, i=1,2$, satisfy conditions (i)-(iv), then series (3.16) converges uniformly in $\bar{Q}$, hence, $u_{1} \in C(\bar{Q})$ and $u_{1}(0, \varphi, z)=0$ because of (3.6). Moreover, in view of the ellipticity of Eq. (1.3) in $Q_{0}$ and due to the condition $c>0$, the sum $u_{1}(r, \varphi, z)$ of series (3.16) is twice continuously differentialable in $Q_{0}$, i.e. it represents the solution of Eq. (1.3) from the class $C^{2}\left(Q_{0}\right) \cap C(\bar{Q})$. Evidently, $u_{1}$ satisfies the boundary value conditions:

$$
u_{1}(r, \varphi,(i-1) h)=f_{i}(r, \varphi), \quad i=1,2,
$$

for $(r, \varphi) \in \bar{\Delta}$, i.e. on the bases $B_{i}$ of cylinder $Q$, and

$$
u_{1}(\rho, \varphi, z)=0
$$

for $(\varphi, z) \in \Omega$, i.e. on the lateral surface $S$ of cylinder $Q$.

If functions $f_{i}, i=1,2$, satisfy conditions (i), (ii), then it follows from (3.17) the uniform convergence of series (3.16) for $r=\delta$, i.e. on the interior lateral surface of cylindrical ring $Q_{\delta}$. According to Lemma 3, series (3.8) converge uniformly in $\bar{\Delta}_{\delta}$. This implies the uniform convergence of series (3.16) on the both bases $B_{i \delta}$ of cylindrical ring $Q_{\delta}$. Therefore, series (3.16) converges uniformly on the whole boundary of cylindrical ring $Q_{\delta}$. Then by similar reasoning as above, we obtain that this series converges uniformly in $\bar{Q}_{\delta}$ and its sum $u_{1}(r, \varphi, z)$ represents the solution of Eq. (1.3) from $C^{2}\left(Q_{0}\right) \cap C\left(\bar{Q}_{\delta}\right)$. Since $\delta$ is whatever, we get that $u_{1} \in C^{2}\left(Q_{0}\right) \cap C(\bar{Q} \backslash\{r=0\})$. Obviously, in this case, $u_{1}$ satisfies condition (3.19), but condition (3.18) is satisfied only for $(r, \varphi) \in \Delta_{0} \cup K$.

Assuming that compatibility condition (1.7) holds, let us consider the solution

$$
u_{2}=u_{1}^{(3)}+u_{1}
$$

of Eq. (1.3), where $u_{1}^{(3)}$ is the obtained above solution of Problem D0 for $\alpha=1$ (see $(2.13))$ and $u_{1}$ is the solution given by (3.16). Note that

$$
u_{2}(\rho, \varphi, z)=f(\varphi, z), \quad(\varphi, z) \in \Omega, \quad u_{2}(r, \varphi,(i-1) h)=f_{i}(r, \varphi), \quad i=1,2,
$$

for each $(r, \varphi) \in \Delta_{0} \cup K$, if functions $f_{i}$ satisfy conditions (i), (ii), and for each $(r, \varphi) \in \bar{\Delta}$, if these functions satisfy conditions (i)-(iv) of Lemma 3. Thus, $u_{2}$ is the solution of Problem D1.

The uniqueness of the solution of Problem D1 follows from Lemma 1.

From reasoning above, we get the following theorem.

Theorem 2. Let $f \in C^{2}(\Omega)$ and let $f_{i}, i=1,2$, satisfy conditions (i), (ii) of Lemma 3. If $\alpha=1$, then there exists the unique solution $u_{2}$ of Problem D1 from the class $C^{2}\left(Q_{0}\right) \cap C(\bar{Q} \backslash\{r=0\})$. If $f_{i}, i=1,2$, satisfy all conditions (i)(iv) of Lemma 3 , then $u_{2} \in C^{2}\left(Q_{0}\right) \cap C(\bar{Q})$. The solution $u_{2}$ can be expressed exactly by (3.20). 


\section{Solution of Problem D (the case $\alpha=1$ )}

Assume that $f \in C^{2}(\Omega), f(\varphi,(i-1) h) \neq 0, i=1,2$, identically, and compatibility condition (1.6) holds. Then, similarly to (2.11), function $f$ can be expanded into uniformly and absolutely converging in $\Omega$ double Fourier series [4]

$$
f(\varphi, z)=\sum_{n=0}^{\infty} a_{0 n} \cos \gamma_{n} z+\sum_{n=1}^{\infty} \sum_{m=1}^{\infty}\left(\alpha_{m n} \cos m \varphi+\beta_{m n} \sin m \varphi\right) \cos \gamma_{n} z
$$

where

$$
\begin{aligned}
& \left.\begin{array}{c}
\alpha_{m n} \\
\beta_{m n}
\end{array}\right\}=\frac{\kappa_{m n}}{\pi^{2}} \int_{0}^{h} \cos \gamma_{n} z \mathrm{~d} z \int_{-\pi}^{\pi} f(\varphi, z)\left\{\begin{array}{ll}
\cos m \varphi \\
\sin m \varphi
\end{array}\right\} \mathrm{d} \varphi, \quad \begin{array}{l}
m, n \in \mathbb{N}_{0}, \\
m \in \mathbb{N}, n \in \mathbb{N}_{0},
\end{array} \\
& \kappa_{00}=1, \quad \kappa_{0 n}=\frac{1}{2}, \quad \kappa_{m n}=\frac{1}{4}, \quad m, n \in \mathbb{N} .
\end{aligned}
$$

By the method of separate variables, let us compose the auxiliary solution $u_{a}$ of Eq. (1.3) by

$$
\begin{aligned}
u_{a}(r, \varphi, z)= & \sum_{n=0}^{\infty} \frac{I_{\sqrt{c}}\left(\gamma_{n} r\right)}{I_{\sqrt{c}}\left(\gamma_{n} \rho\right)} \alpha_{0 n} \cos \gamma_{n} z \\
& +\sum_{n=0}^{\infty} \sum_{m=1}^{\infty} \frac{I_{\sqrt{m^{2}+c}}\left(\gamma_{n} r\right)}{I_{\sqrt{m^{2}+c}}\left(\gamma_{n} \rho\right)}\left(\alpha_{m n} \cos m \varphi+\beta_{m n} \sin m \varphi\right) \cos \gamma_{n} z,
\end{aligned}
$$

which satisfies the evident condition

$$
u_{a}(\rho, \varphi, z)=f(\varphi, z), \quad(\varphi, z) \in \Omega .
$$

(The convergence of series (4.2) to the solution of Eq. (1.3) and inclusion $u_{a} \in$ $C^{2}\left(Q_{0}\right) \cap C(\bar{Q})$ can be justified just in the same way as in the case of series $(2.13))$.

Lemma 4. Let $f \in C^{2}(\Omega)$. Then $\frac{\partial u_{a}}{\partial \varphi} \in C(\Omega)$ and

$$
\frac{\partial^{k} u_{a}(r, \varphi, z)}{\partial r^{k}}=O\left(r^{\sqrt{c}-k}\right) \quad \text { as } r \rightarrow 0, k=0,1,2,
$$

uniformly in $Q$.

Proof. Due to the assumption $f \in C^{2}(\Omega)$, series (3.20) is term-by-term differentiable with respect to $\varphi$, i.e.

$$
\frac{\partial f(\varphi, z)}{\partial \varphi}=\sum_{n=1}^{\infty} \sum_{m=1}^{\infty} m\left(-\alpha_{m n} \cos m \varphi+\beta_{m n} \sin m \varphi\right) \cos \gamma_{n} z,
$$

besides, the series on right-hand side converges uniformly and absolutely in $\Omega$. Thus,

$$
\sum_{n=1}^{\infty} \sum_{m=1}^{\infty} m\left(\left|\alpha_{m n}\right|+\left|\beta_{m n}\right|\right)<\infty
$$


By differentiation of series (4.2) with respect to $\varphi$, we obtain that

$$
\frac{\partial u_{a}(r, \varphi, z)}{\partial \varphi}=\sum_{n=1}^{\infty} \sum_{m=1}^{\infty} \frac{I_{\sqrt{m^{2}+c}}\left(\gamma_{n} r\right)}{I_{\sqrt{m^{2}+c}}\left(\gamma_{n} \rho\right)} m\left(-\alpha_{m n} \cos m \varphi+\beta_{m n} \sin m \varphi\right) \cos \gamma_{n} z .
$$

Since functions $I_{\sqrt{m^{2}+c}}\left(\gamma_{n} r\right), m \in \mathbb{N}_{0}$, are monotonically decreasing on the interval $(0, \rho)[13]$, we get that

$$
\left|\frac{\partial u_{a}(r, \varphi, z)}{\partial \varphi}\right| \leq \sum_{n=1}^{\infty} \sum_{m=1}^{\infty} m\left(\left|\alpha_{m n}\right|+\left|\beta_{m n}\right|\right)<\infty
$$

in $(r, \varphi, z) \in Q$. Thus the first assertion of the lemma is true.

Further, it follows from asymptotic properties of modified Bessel functions $[3,13]$ that

$$
\frac{\mathrm{d}^{k}}{\mathrm{~d} r^{k}} I_{\sqrt{m^{2}+c}}\left(\gamma_{n} r\right)=\kappa_{k}\left(\frac{\gamma_{n}}{2} r\right)^{\sqrt{m^{2}+c}-2}\left(1+O\left(r^{2}\right)\right), \quad k=0,1,2,
$$

as $r \rightarrow 0, m, n \in \mathbb{N}_{0}$, where $\kappa_{0}=1$ and $\kappa_{k}=\frac{1}{2}$ for $k=1,2$. According to this, we obtain from (4.2) that

$$
\lim _{r \rightarrow 0} r^{k-\sqrt{c}} \frac{\partial^{k} u_{a}(r, \varphi, z)}{\partial r^{k}}=2^{-\sqrt{c}} \sum_{n=0}^{\infty} \frac{\gamma_{n}^{\sqrt{c}-k}}{I_{\sqrt{c}}\left(\gamma_{n} \rho\right)} \alpha_{0 n} \cos \gamma_{n} z, \quad k=0,1,2 .
$$

Since

$$
\frac{\gamma_{n}^{\sqrt{c}-1}}{I_{\sqrt{c}}\left(\gamma_{n} \rho\right)} \rightarrow 0 \quad \text { as } n \rightarrow \infty
$$

(because of asymptotic properties of Bessel function $I_{\sqrt{c}}(t)$ as $t \rightarrow+\infty$ [3]), the absolute convergence of series (4.1) yields the absolute convergence of the series on the right of the last equality. Therefore, assertion (4.4) is also true.

Introduce the functions $g_{i}, i=1,2$, by

$$
g_{i}(r, \varphi):=u_{a}((r, \varphi,(i-1) h))-f_{i}(r, \varphi),
$$

where $u_{a}$ is given by (4.2). Observe that

$$
g_{i}(\rho, \varphi)=f(\varphi,(i-1) h)-f_{i}(\rho, \varphi)=0
$$

because of (1.7).

Directly from Lemma 4, we obtain the following corollary.

Corollary. Let compatibility condition (1.7) holds. If $f \in C^{2}(\Omega)$ and the functions $f_{i}, i=1,2$, satisfy conditions (i), (ii) of Lemma 3, then the functions $g_{i}, i=1,2$, also satisfy the same conditions. If, in addition, the functions $f_{i}$, $i=1,2$, satisfy conditions (iii), (iv) of Lemma 4 and the inequality $c \geq 4$ holds, then the functions $g_{i}, i=1,2$, satisfy all conditions ((i)-(iv) of Lemma 3 . 
Theorem 3. Let $f \in C^{2}(\Omega)$ and let compatibility condition (1.7) holds. If functions $f_{i}, i=1,2$, satisfy conditions (i), (ii) of Lemma 3, then there exists the solution of Problem D from the class $C^{2}\left(Q_{0}\right) \cap C(\bar{Q} \backslash\{r=0\})$. If functions $f_{i}, i=1,2$, are such that all conditions ( $(\mathrm{i})-(\mathrm{iV})$ are satisfied and if $c \geq 4$, then Problem $\mathrm{D}$ has the solution from the class $C^{2}\left(Q_{0}\right) \cap C(\bar{Q})$. In both cases the solution is unique.

Proof. Let us consider the following Problem D1 to Eq. (1.3):

$$
\begin{aligned}
& u(\rho, \varphi, z)=0, \quad(\varphi, z) \in \Omega, \\
& u((r, \varphi,(i-1) h))=g_{i}(r, \varphi), \quad i=1,2,
\end{aligned}
$$

where functions $g_{i}$ are defined by (4.5). According to Theorem 2 and to Corollary, there exists the unique solution $u_{1}^{(0)}(r, \varphi, z)$ of problem $(4.6),(4.7)$ such that

a) $u_{1}^{(0)} \in C^{2}\left(Q_{0}\right) \cap C(\bar{Q} \backslash\{r=0\})$ and boundary value condition (4.7) is satisfied for $(r, \varphi) \in \Delta_{0} \cup K$, if functions $f_{i}, i=1$, 2 , satisfy conditions (i), (ii) of Lemma 3;

b) $u_{1}^{(0)} \in C^{2}\left(Q_{0}\right) \cap C(\bar{Q})$ and boundary value condition (4.7) is satisfied for each in $(r, \varphi) \in \bar{\Delta}$, if functions $f_{i}, i=1,2$, satisfy conditions (i)-(iv) of Lemma 3 and if $c \geq 4$.

Besides, the solution $u_{1}^{(0)}$ can be obtained analytically in the same way as the solution $u_{1}$ (see (3.16)) of the identically problem with boundary value condition (1.5).

Introduce the following solution of Eq. (1.3):

$$
u=u_{a}-u_{1}^{(0)}
$$

where the component $u_{a}$ is given by (4.2). Since $u_{a} \in C^{2}\left(Q_{0}\right) \cap C(\bar{Q})$ under condition $f \in C^{2}(\Omega)$, solution (4.8) is from the same class as the solution $u_{1}^{(0)}$ of problem $(4.6),(4.7)$. It is easily seen that

$$
u(\rho, \varphi, z)=f(\varphi, z), \quad(\varphi, z) \in \Omega,
$$

(in view of (4.3) and (4.6)) and

$$
u(r, \varphi,(i-1) h)=f_{i}(r, \varphi), \quad i=1,2,
$$

(due to definition (4.5)) for $(r, \varphi) \in \Delta_{0} \cup K$, if functions $f_{i}, i=1,2$, satisfy conditions (i), (ii), and for $(r, \varphi) \in \bar{\Delta}$, if both $f_{i}$ satisfy conditions (i)-(iv) of Lemma 3. Thus, the boundary value conditions (1.4), (1.5) are satisfied and, consequently, (4.8) represents the solution of Problem D.

Since $u_{a}(0, \varphi, z)=u_{1}^{(0)}(0, \varphi, z)=0$, the uniqueness of solution $u$ of Problem $\mathrm{D}$ follows from Lemma 1.

\section{Conclusions}

1. The continuity of the solution of Problem D on the line of the degeneracy depends on the behavior of the given boundary functions in the vicinity of the points $P_{0}$ and $P_{h}$, in which this line crosses the bases of cylinder $Q$. 
2. If the boundary functions being continuous on the surface of cylinder $Q$ are not identically equal to zero on the both edges of this cylinder, then the continuity of the solution of Problem D depends on the properties of boundary functions, but also on the coefficient $c$ of considered equation.

\section{References}

[1] D. Gilbarg and N. Trudinger. Elliptic Partial Differential Equations. Springer, Berlin, 1983.

[2] V.V. Katrakhov and S.M. Sitnik. A boundary value problem of the steady-stay Schroedinger equation with a singular potential. Sowiet Math. Dokl., 30(2):468470, 1984.

[3] A. Kratzer and W. Franz. Transcendente funktionen. Akad. Verlagsgesellshaft, Leipzig, 1960.

[4] F. Moricz and A. Veres. Absolute convergence of multiple Fourier series revisited. Anal. Math., 34:145-162, 2008. https://doi.org/10.1007/s10476-008-0205-7.

[5] F.W.J. Olver. Asymptotics and Special Functions. Academic Press, New York, 1974.

[6] S. Rutkauskas. Weighted boundary problems for a second-order ordinary differential equation with singularity. I. Lith. Math. J., 26(4):367-376, 1986. https://doi.org/10.1007/BF00971919.

[7] S. Rutkauskas. The Dirichlet type problem with asymptotic conditions for an elliptic system degenerating at a point, I. Differ. Equations, 38(3):405-412, 2002. https://doi.org/10.1023/A:1016070111630.

[8] S. Rutkauskas. The Dirichlet type problem with asymptotic conditions for elliptic systems degenerating at a point, II. Differ. Equations, 38(5):719-725, 2002. https://doi.org/10.1023/A:1020223027249.

[9] S. Rutkauskas. Exact solutions of the Dirichlet problem to the degenerate a line elliptic system. Doklady AMAN, 17(4):84-88, 2015.

[10] S. Rutkauskas. Exact solutions of dirichlet type problem to elliptic equation, which type degenerates at the axis of cylinder. I. Boundary Value Probl., 183, 2016. https://doi.org/10.1186/s13661-016-0690-8.

[11] S. Rutkauskas. Exact solutions of Dirichlet type problem to elliptic equation, which type degenerates at the axis of cylinder. II. Boundary Value Problems, 182, 2016. https://doi.org/10.1186/s13661-016-0691-7.

[12] G.P. Tolstov. Fourier Series. Dover, New York, 1976.

[13] G.N. Watson. A Treatise on the Theory of Bessel Functions. Cambridge University Press, Cambridge, 1995.

[14] A. Yanushauskas. Analytical theory of the elliptic equations. Nauka (Siberian branch), Novosibirsk, 1979 (in Russian). 